\title{
RECURRENT TEMPOROMANDIBULAR JOINT DISLOCATIONS IN GERIATRIC PATIENT ON ANTIPSYCHOTIC DRUGS AND ITS CONSERVATIVE MANAGEMENT
}

\author{
DEEPIKA PAI ${ }^{1 *}$, ABHAY T KAMATH ${ }^{2}$, GIRISH MENON $^{3}$, ARUN URALA $^{4}$, SAURABH KUMAR $^{1}$, SUNIL NAYAK $^{2}$, \\ ADARSH KUDVA ${ }^{2}$
}

${ }^{1}$ Department of Pedodontics and Preventive Dentistry, Manipal College of Dental Sciences, Manipal University, Manipal, Karnataka,

India. ${ }^{2}$ Department of Oral and Maxillofacial Surgery, Manipal College of Dental Sciences, Manipal, Karnataka, India. ${ }^{3}$ Department of Neurosurgery, Kasturba Medical College, Manipal University, Manipal, Karnataka, India. ${ }^{4}$ Department of Orthodontics, Manipal College of Dental Sciences, Manipal University, Manipal, Karnataka, India. Email: deepikapai0479@gmail.com

Received: 16 June 2017, Revised and Accepted: 08 August 2017

ABSTRACT

Recurrent temporomandibular joint (TMJ) dislocation can cause difficulty in swallowing and speech hence can be distressing for the patient. A 79-year-old male patient reported with recurrent dislocation of TMJ since 2 months. He was on antipsychotic medication for schizophrenia which predisposes to recurrent TMJ dislocation due to oromandibular dystonia. Since the patient had undergone tracheostomy, his systemic condition was not suitable for surgical management. Thus a conservative option of chin cup was planned. The chin cup limits the movement of the mandible hence prevents dislaocation of the TMJ. The patient was successfully rehabilitated with no new episodes of TMJ dislocation for the last 4 months. It focuses on conservative management of recurrent TMJ dislocations induced by antipsychotic drugs.

Keywords: Chin cup appliance, Geriatric patient, Recurrent temporomandibular joint dislocation, Antipsychotic drugs.

(c) 2017 The Authors. Published by Innovare Academic Sciences Pvt Ltd. This is an open access article under the CC BY license (http://creativecommons. org/licenses/by/4. 0/) DOI: http://dx.doi.org/10.22159/ajpcr.2017.v10i11.20748

\section{INTRODUCTION}

Recurrent temporomandibular joint (TMJ) dislocation refers to persistent luxation of the TMJ that occurs during daily activities like yawning and laughing [1]. Several factors associated with it are trauma, drugs like phenothiazine and metoclopramide, systemic conditions like connective tissue disorders and neuromuscular disorders [2]. They usually present with complaints of the inability to chew and swallow, difficulty in speaking, drooling of saliva. The treatment of recurrent TMJ dislocation can be accomplished by conservative or surgical approaches. Conservative options to manage recurrent TMJ dislocation vary from maxillomandibular fixation, elastic bandaging, and even injecting sclerosing agents or botulin toxins $[3,4]$. We present a case of recurrent dislocation of the TMJ in a 79-year-old male on the antipsychotic drug for the treatment of schizophrenia, where the patient's systemic condition and medication status were not favorable towards surgical management and hence the conservative management using chin cup appliance was done.

\section{CASE REPORT}

A 79-year-old male patient was referred to the Department of Oral and Maxillofacial Surgery with the chief complaint of inability to close the mouth and difficulty in swallowing and talking. On interviewing the patient family, they revealed that this incident happened during chewing of food. They also reported that this kind of dislocation frequently occurred in the last 2 months and the patient had to be rushed to a hospital for reducing the mandible in every such event. The patient was a known case of hypertension and diabetes. Adding further in medical history the patient revealed that he had fallen 3 months back, which led to bleeding of left parietal basal ganglia which was evacuated by left temporoparietal craniotomy by neurosurgeons. Following which he developed bronchopulmonary ventilator-associated pneumonia for which tracheostomy was done. He also was taking tablet Quitipin for the treatment of schizophrenia since the last 2 months.

On examination, the patient had a leptoprosopic face with jaw deviated to the left side (Fig. 1). Intraoral examination revealed that the patient was partially edentulous but was not wearing any prosthesis, had thus exhibited deep bite. On palpation, the TMJ and masticatory muscles were not tender. Bilateral depression was found in the preauricular region with the condyle being palpable $3 \mathrm{~cm}$ in front of the depression.

Based on the history and clinical examination the diagnosis of chronic bilateral recurrent TMJ dislocation was derived. Mandible was reduced by Hippocratic method (Fig. 2). A chin cup was delivered as a restrainer (Fig. 3). The chin cup was fitted on the chin, and the head strap was secured around the head after adjusting the force element to a position to allow comfortable movements of the jaw also at the same time prevent further dislocation. Thus, the chin cup acts as a passive restraining appliance. The patient was instructed to wear the appliance continuously throughout the daily activities. The patient was followed up after 7 days and did not report any pain or tenderness in the TMJ region or the masticatory muscles. The patient was comfortable in wearing the appliance and also did not report of a new episode of dislocation thereafter for 4 months.

\section{DISCUSSION}

TMJ dislocations are reported in the age range from 10 to 79 years, but recurrent dislocations are highly associated with geriatric patients [5]. This is perhaps owing to the age-related changes of articular surfaces, loss of laxity of ligaments surrounding the joint capsule, loss of neuromuscular coordination as in our case $[6,7]$.

Several drugs such as phenothiazine, metoclopramide, and antipsychotics have also been associated with recurrent TMJ dislocations $[1,8]$. Quitipin is one such antipsychotic drug which can cause recurrent TMJ dislocations. The drug-induced TMJ dislocations are secondary to oromandibular dystonia and oral muscular dyskinesia caused due to extrapyramidal effects of the drug [8-10]. A few reports on TMJ dislocations due to antipsychotic drugs do exist in the literature [8,11-13]. These reports have opted the use of nonsurgical interventions like the use of botulin toxins to reduce the TMJ dislocations [12]. Since such TMJ dislocation is a possible 


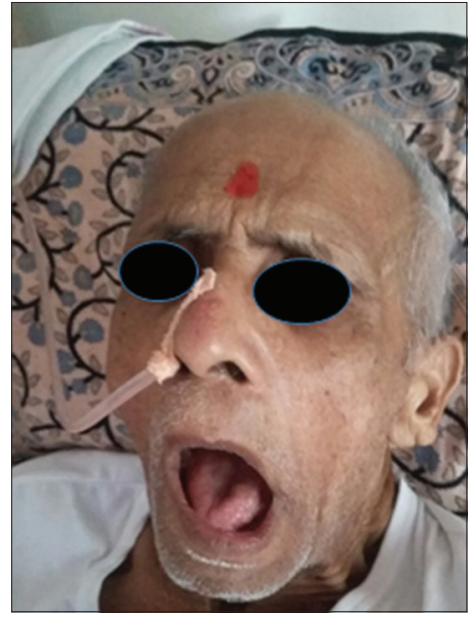

Fig. 1: Patient showing dislocated temporomandibular joint with jaw deviated to the left side

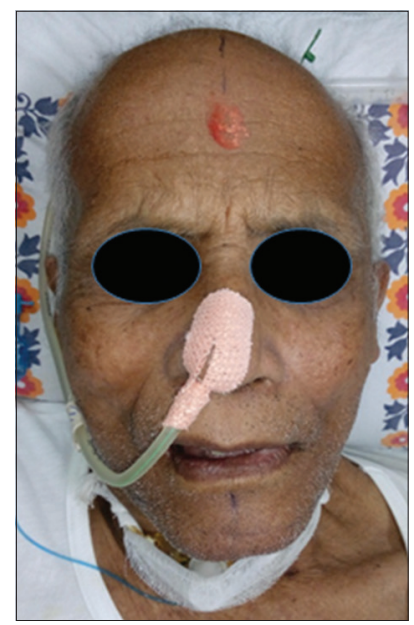

Fig. 2: Patient with temporomandibular joint dislocation that is reduced by Hippocratic method

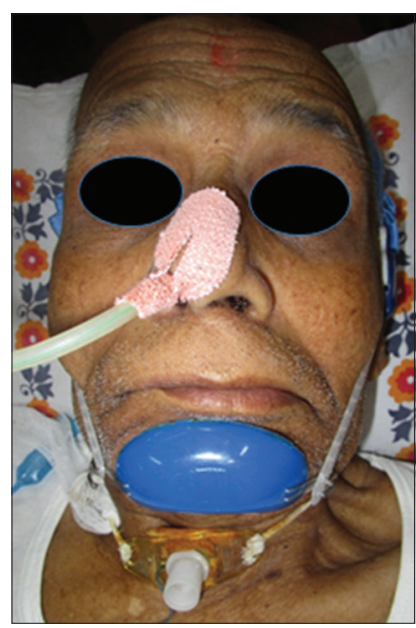

Fig. 3: Patient wearing chin cup

complication of the drug, nonsurgical modalities of treatment are most justified in these cases. In our case, the patient was on Quitipin since 2 months. Surgical treatment of recurrent dislocation of TMJ is viewed with overwhelming success compared to conservative nonsurgical options $[14,15]$. However, surgical treatment is not suitable for all patients. As in our case the age of the patient, his compromised systemic conditions were not suitable for surgical intervention neither could the medication be stopped owing to his medical condition, hence a more conservative, non-invasive treatment modality of chin cup appliance was planned. Due to age considerations, various conservative management approaches have been employed in the management of orofacial lesions especially in children as well as geriatric patients [16].

Nonsurgical methods of managing recurrent TMJ dislocations include elastic bandaging, chin cap and injection of various sclerosing agents such as alcohol, sodium tetradecyl sulfate, sodium psylliate, morrhuate sodium, and platelet-rich plasma into the joint space [17]. Injection of autologous blood into the joint space is also a nonsurgical alternative in the treatment of recurrent TMJ dislocations [2]. All the above agents cause an increase in fibrosis in the upper joint space, the pericapsular tissues, or both and produce restriction in condylar movements thus prevent further dislocations. Besides injection of sclerosing agents is viewed as an invasive treatment modality, several anecdotal reports suggest that this fibrosing effect is temporary [18]. Hence, we resorted to a conservative non-invasive technique of restraining the condyle in the appropriate position with the use of chin cup appliance.

The chin cup appliance consists of a chin cup that is secured with the help head strap. This, when placed over the chin of patient after reducing the dislocated TMJ, prevents further episodes of dislocation by restraining the movement of the mandible beyond the physiologic limits. The securing straps are adjusted to provide the only optimal opening of the mouth and prevent excessive opening that can cause dislocation of TMJ. The patient was instructed to wear the chin cup continuously throughout the day and carry out routine activities with the appliance in place. Chin cup, when used as an orthodontic appliance actively, exerts a restrictive force on the growing prognathic mandible, but when chin cup is given to prevent the TMJ dislocation, it passively restrains the mandible. Our patient did not report any pain or tenderness in the TMJ area or masticatory muscles during follow-up. The patient compliance toward the chin cup appliance was favorable, and our patient has not reported of a new episode of dislocation since last 4 months.

Oral health-related problems can be alternatively managed with regularly available appliances or with minor modifications of the same. The routinely used trainers have been used to intercept habits along with malocclusion [19]. Similarly, yet another myofunctional appliance like chin cup is put to utilization in a geriatric patient to restrain the mandible in our patient.

Oral health problems can significantly reduce the quality of life of any individuals with compromised health, especially in pediatric and geriatric patients [20]. Conservative approaches can suitably improve the well-being of such individual.

\section{CONCLUSION}

The chin cup appliance is known to all dentists as a device for correction of malocclusion, but it can also be used as a preventive, restraining appliance in patients with recurrent TMJ dislocation especially in those who are unfit of surgical intervention of the TMJ.

We also report this case with interest to highlight that the recurrent TMJ dislocation can occur commonly in patients on antipsychotic medications. Hence, one needs to be alarmed of such adverse outcomes of prescribing these drugs, so as to take necessary preventive steps. It also is not convincing to terminate the antipsychotic drugs in these patients. The article highlights a conservative management of recurrent TMJ dislocation in these patients.

\section{REFERENCES}

1. Yoshioka N, Shimo T, Ibaragi S, Sasaki A. Autologous blood injection for the treatment of recurrent temporomandibular joint dislocation. Acta Med Okayama 2016;70(4):291-4. 
2. Marqués-Mateo M, Puche-Torres M, Iglesias-Gimilio ME. Temporomandibular chronic dislocation: The long-standing condition. Med Oral Patol Oral Cir Bucal 2016;21(6):e776-83.

3. Matsushita K, Abe T, Fujiwara T. OK-432 (Picibanil) sclerotherapy for recurrent dislocation of the temporomandibular joint in elderly edentulous patients: Case reports. $\mathrm{Br} \mathrm{J}$ Oral Maxillofac Surg 2007;45(6):511-3.

4. Fu KY, Chen HM, Sun ZP, Zhang ZK, Ma XC. Long-term efficacy of botulinum toxin type a for the treatment of habitual dislocation of the temporomandibular joint. Br J Oral Maxillofac Surg 2010;48(4):281-4.

5. Chhabra S, Chhabra N, Gupta P Jr. Recurrent mandibular dislocation in geriatric patients: Treatment and prevention by a simple and noninvasive technique. J Maxillofac Oral Surg 2015;14 Suppl 1:231-4.

6. Mathew AL, Sholapurkar AA, Pai KM. Condylar changes and its association with age, TMD, and dentition status: A cross-sectional study. Int J Dent 2011;2011:413639.

7. Magnusson C, Nilsson M, Magnusson T. Degenerative changes of the temporomandibular joint. Relationship to ethnicity, sex and occlusal supporting zones based on a skull material. Acta Odontol Scand 2012;70(3):207-12.

8. de Araújo AN, do Nascimento MA, de Sena EP, Baptista AF. Temporomandibular disorders in patients with schizophrenia using antipsychotic agents: A discussion paper. Drug Healthc Patient Saf 2014;6:21-7.

9. Anton JM, Ivanova SA. Role of 5-HT2C receptors in dyskinesia. Int J Pharm Pharm Sci 2016;8(1):5-10.

10. Ilyaz M, Baig MM, Quadir MA, Fathima M, Khan SA. Drug utilization study of antipsychotics and its common ADR'S in the psychiatry OPD of OHRC. Int J Pharm Pharm Sci 2014;6(9):162-5.
11. Karthik MS, Prabhu N. Temporomandibular joint dislocation due to atypical antipsychotic-induced acute dystonia: A case report. Ther Adv Psychopharmacol 2014;4(6):282-4

12. Ibrahim ZY, Brooks EF. Neuroleptic-induced bilateral Temporomandibular joint dislocation. Am J Psychiatry 1996;153(2):293-4.

13. Sankhla C, Lai EC, Jankovic J. Peripherally induced oromandibular dystonia. J Neurol Neurosurg Psychiatry 1998;65(5):722-8

14. Jeyaraj P, Chakranarayan A. A conservative surgical approach in the management of longstanding chronic protracted Temporomandibular joint dislocation: A case report and review of literature. J Maxillofac Oral Surg 2016;15 Suppl 2:361-70.

15. Undt G, Kermer C, Rasse M. Treatment of recurrent mandibular dislocation, Part II: Eminectomy. J Craniomaxillofac Surg 1996;24(3):184-8

16. Pai D, Kamath AT, Kudva A, Solomon MM, Kumar S, Sasikumar P. Concomitant central giant cell granuloma and aneurysmal bone cyst in a young child. Case Rep Dent 2017;2017:6545848.

17. Sharma NK, SinghAK, PandeyA, Verma V, Singh S. Temporomandibular joint dislocation. Natl J Maxillofac Surg 2015;6(1):16-20.

18. Ziegler CM, Haag C, Mühling J. Treatment of recurrent Temporomandibular joint dislocation with intramuscular botulinum toxin injection. Clin Oral Investig 2003;7:52-5.

19. Pai D, Kumar S, Kamath AT, Bhaskar V. Pernicious effects of toe sucking habit in children. Case Rep Dent 2016;2016(1):2475784.

20. Kumar S, Pai D, Saran R. Oral health characteristics and dental rehabilitation of children with global developmental delay. Case Rep Dent 2017;2017:5486327. 